\title{
Phenylbutyrate inhibits Diet induced Obesity through inhibition of pyruvate dehydrogenase kinase
}

\author{
DONGWOOK KIM¹, Byoung-keol Min², HYEON-JI KANG², Byoungjun Choi², IN-KYU \\ LEE $^{3}$ \\ ${ }^{1}$ Leading-edge Research Center for Drug Discovery and Development for Diabetes and Metabolic Disease, Kyungpook National \\ University Medical Center, Daegu, Korea \\ 2 Department of Biomedical Science, Kyungpook National University Graduate School, Daegu, Korea \\ ${ }^{3}$ Department of Internal Medicine, Kyungpook National University School of Medicine, Daegu, Korea
}

\section{INTRODUCTION and OBJECTIVES}

Obesity caused by excess energy intake has been emerging as a major health concern in the world, with increased risk leading to diabetes, hypertension, nephropathy, and cardiovascular disease A better understanding of the molecular mechanism on how obesity develops is of critically clinical importance. Accumulating data suggest that ER-stress is strongly related with development of obesity, implying that ER stress can be a therapeutically target of obesity. 4-Phenylbutyrate (PBA) which is known as a chemical chaperon has known to decrease ER-stress. However, the detailed molecular mechanism by which PBA decreases ERstress remains elusive. In this study, we examine the effect of PBA on diet-induced obesity.

\section{METHODS}

\section{Animal study :}

Diet induced obesity (DIO) model. Ob/Ob model

For DIO model, C57/BL6 mice were fed High fat diet (60\% fat) for 8 weeks and were treated with PBA $(1 \mathrm{~g} / \mathrm{kg})$ and vehicle control (PBS) for 8 weeks.

For $\mathrm{Ob} / \mathrm{Ob}$ mice, 8 weeks old mice were treated with $\mathrm{PBA}(1 \mathrm{~g} / \mathrm{kg})$ and vehicle control (PBS) for 5 weeks

18-FDG uptake

C57BL6/J ( 8 weeks) were fed HFD (60\% fat) for 8 weeks then administered with or without PBA ( $1 \mathrm{~g} / \mathrm{L}$ ) for 1 weeks. Glucose uptake was measured by PET/CT.

\section{RESULTS}

A

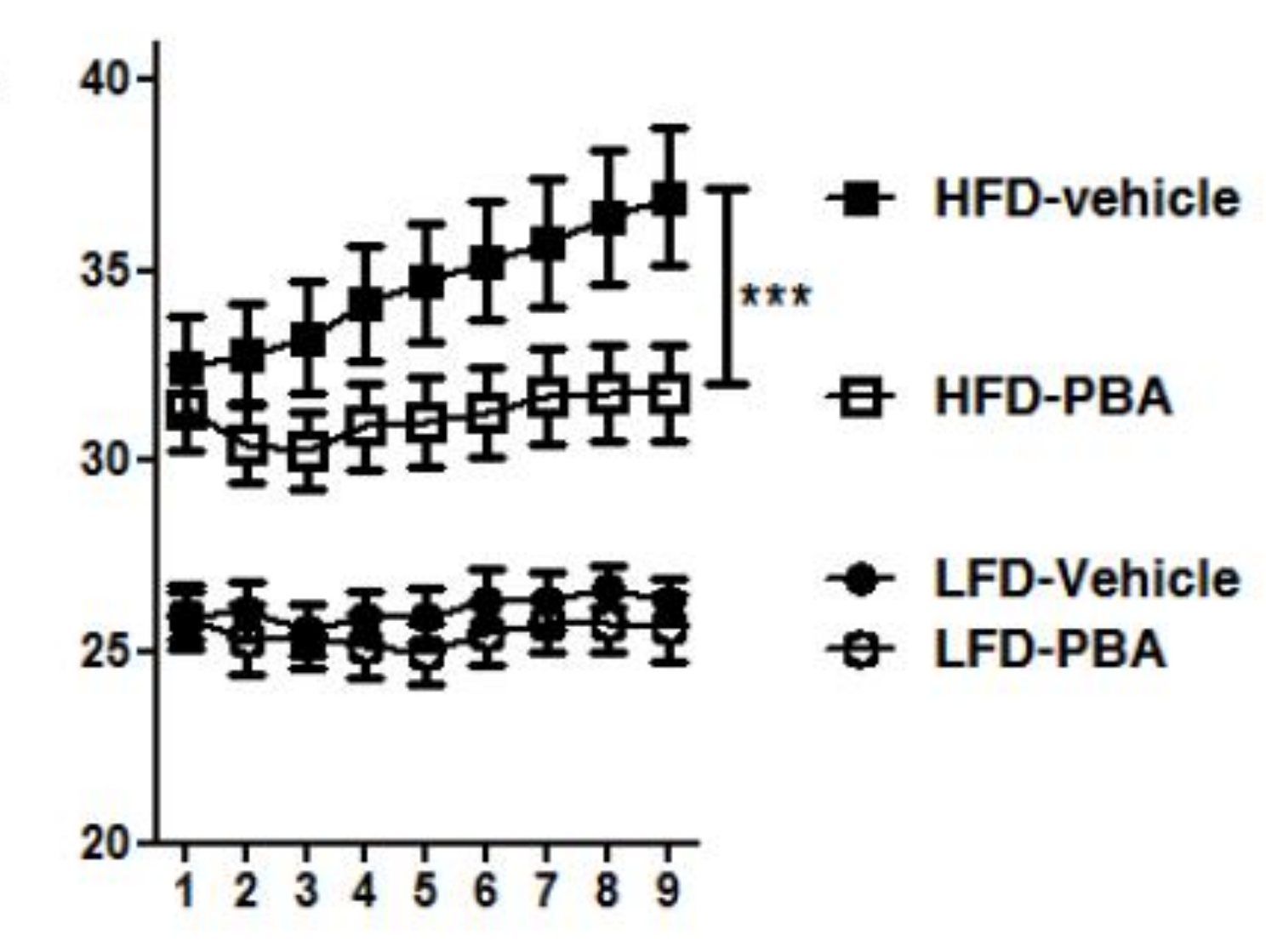

$\mathrm{C}$

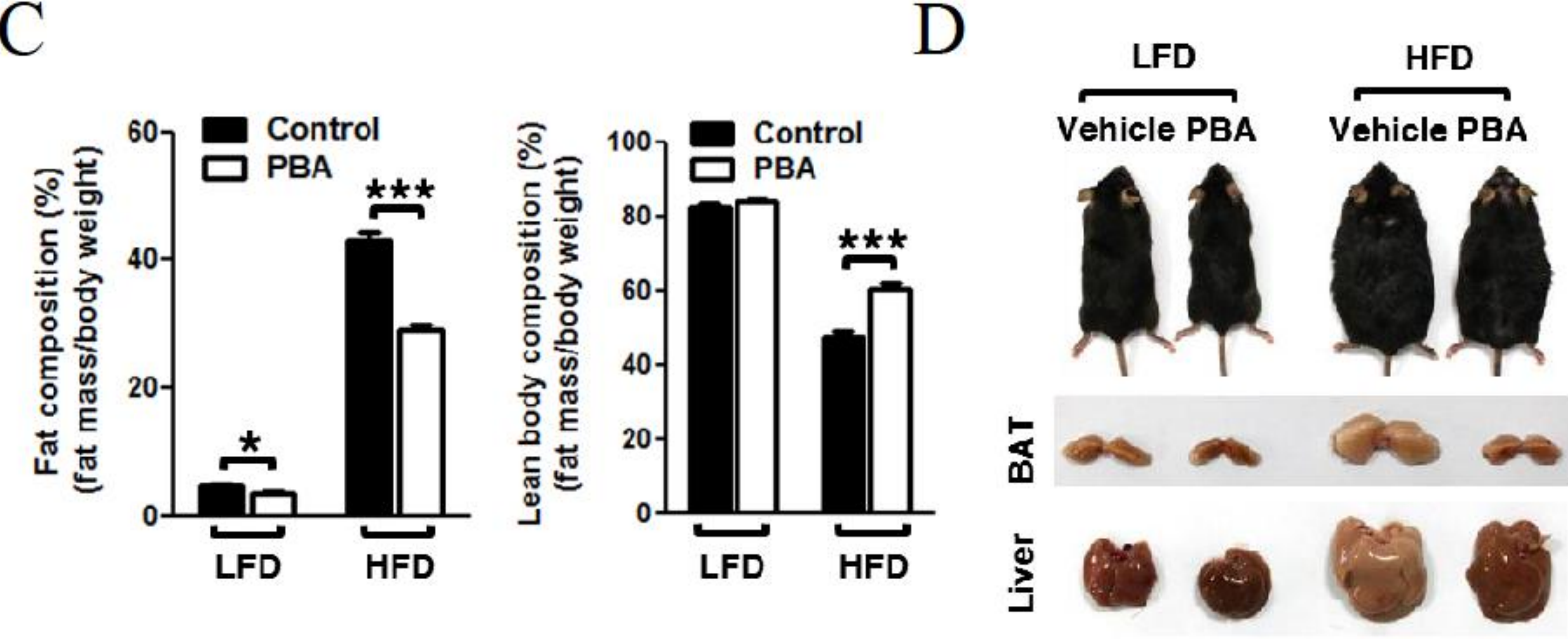

Figure 1. PBA ameliorate HFD induced obesity

(A) PBA decrease Body weight gain by HFD $(n=10)$

(B) CT scan identify PBA decrease Fat accumulation Blue color indicates Fat

(C) Tissue composition analysis showed that PBA decreased Fat mass

(D) Fatty Liver and BAT by HFD is recovered to normal phenotype by PBA

\section{RESULT}

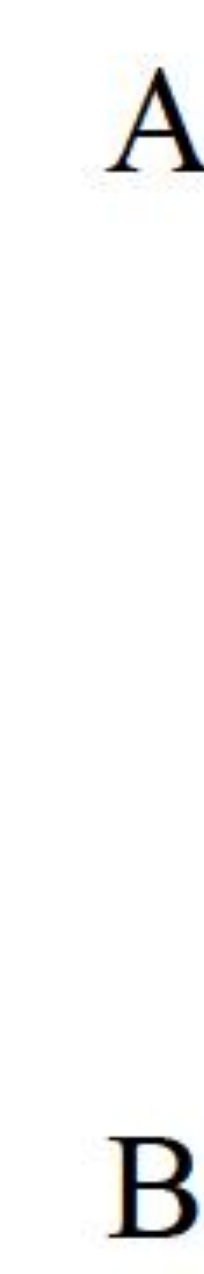

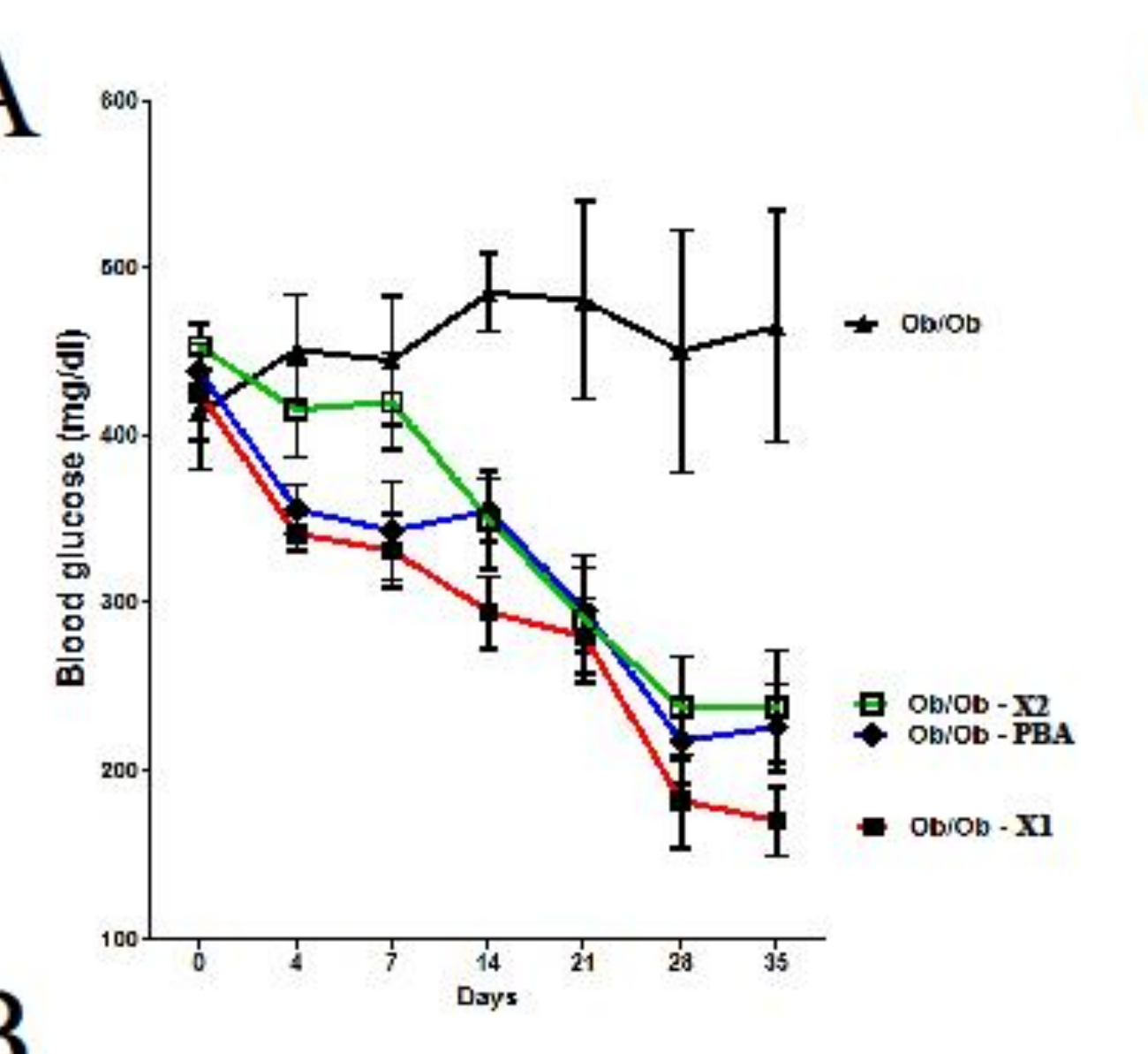
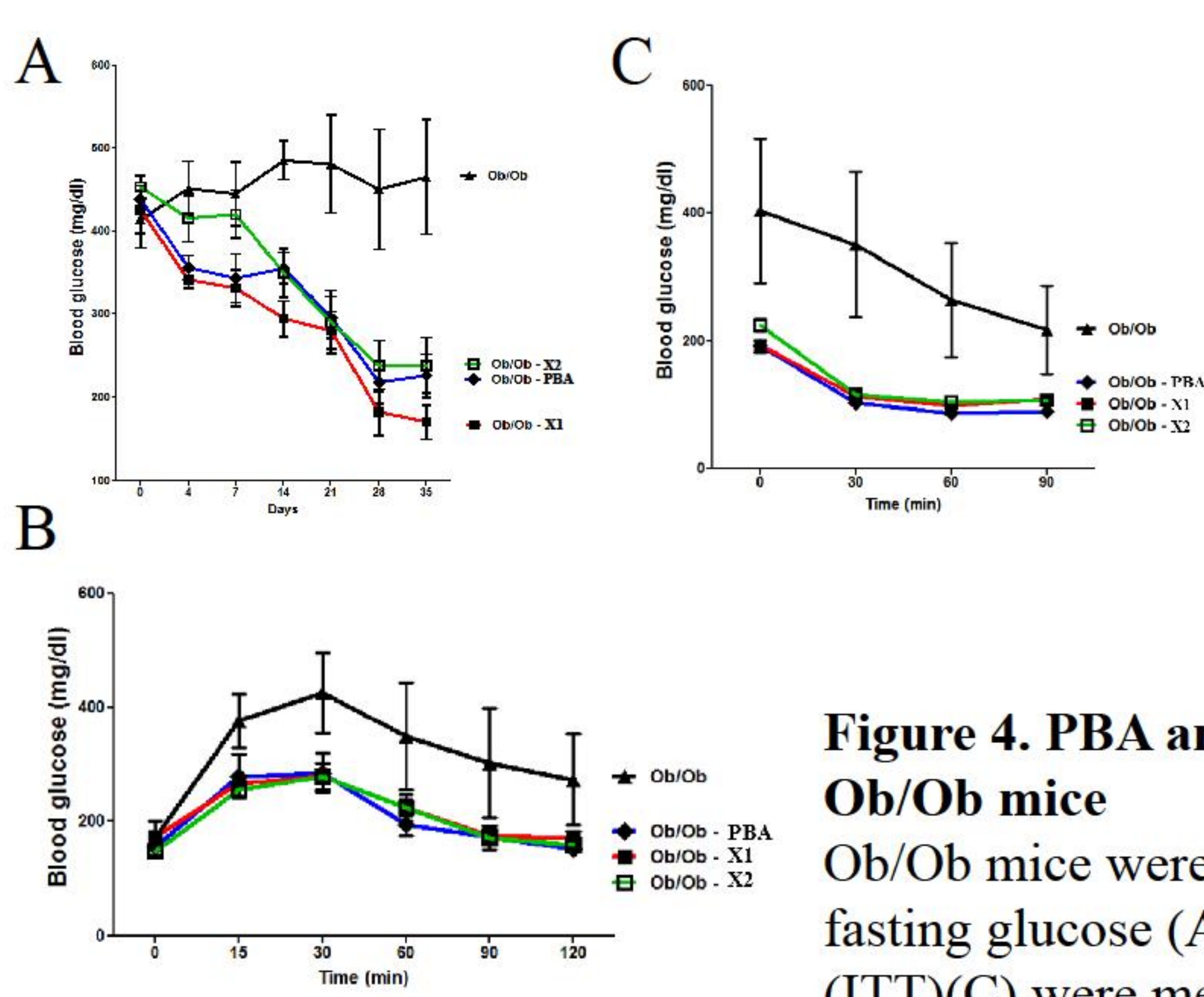

Figure 4. PBA and its derivatives improve metabolic phenotype in $\mathrm{Ob} / \mathrm{Ob}$ mice

$\mathrm{Ob} / \mathrm{Ob}$ mice were treated with PBA and PBA derivatives $(1 \mathrm{~g} / \mathrm{kg})$ and fasting glucose (A)Glucose tolerance test (GTT)(B), insulin tolerance test (ITT)(C) were measured.

(D) Liver tissue treated with PBA and its derivatives was subjected to western analysis

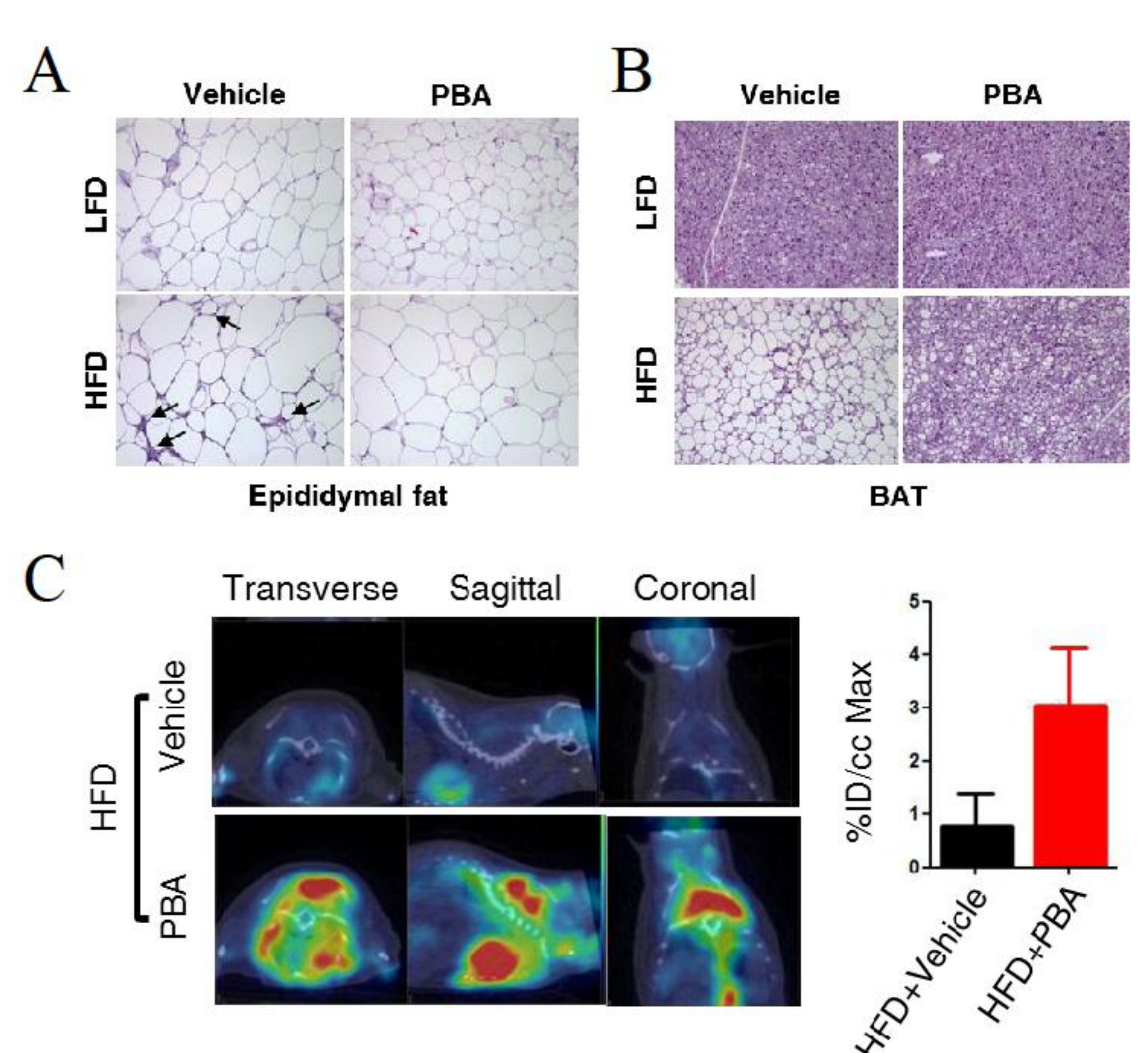

Figure 2. PBA improve Fat activities

(A) PBA decrease macrophage infiltration by HFD in White adipose tissue (WAT)

(B) PBA decreased fat accumulation in Brown adipose tissue (BAT)

(C) 18-FDG uptake identified that PBA increase glucose uptake in BAT

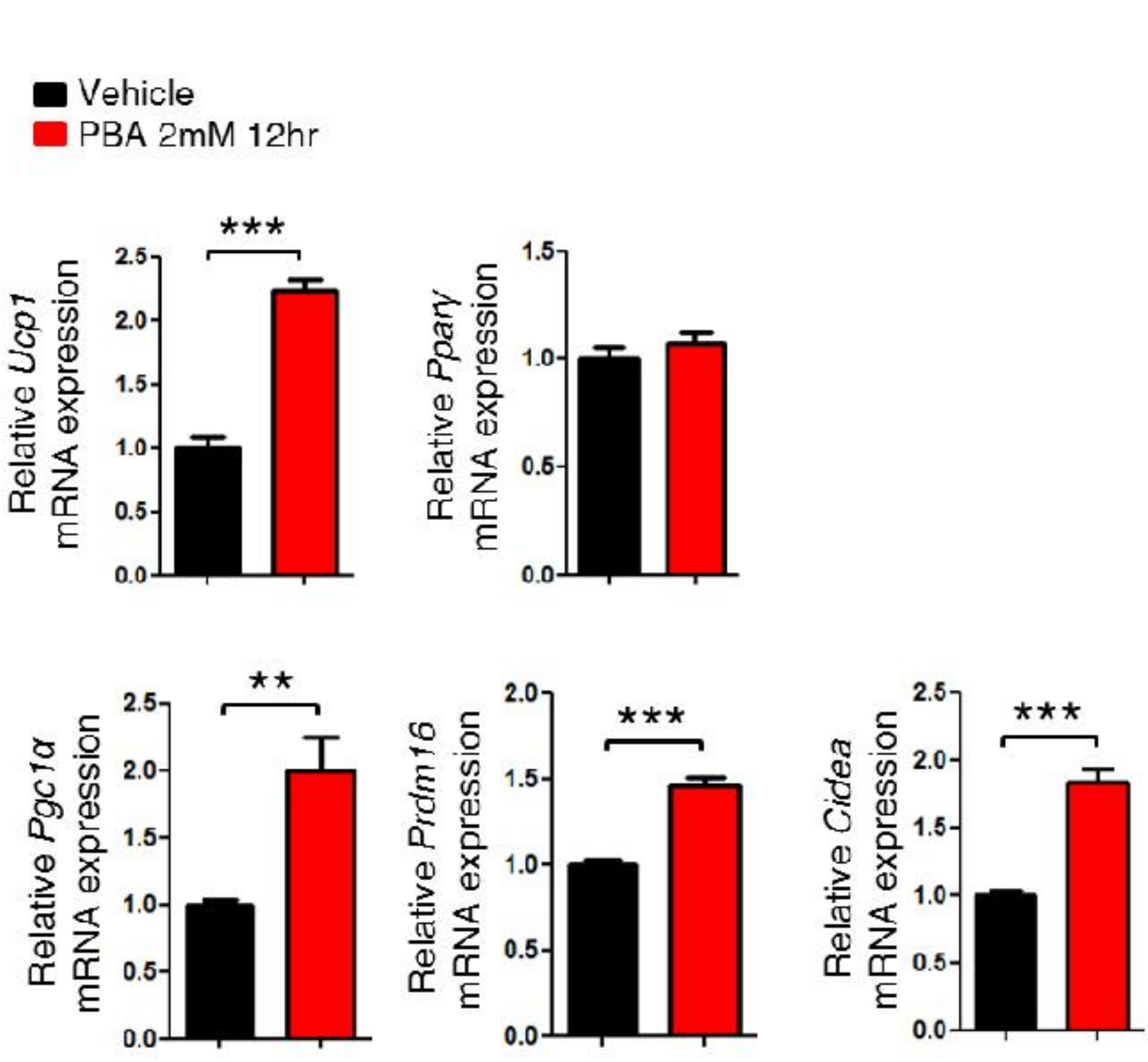

Figure 3. PBA increase genes expression related with $B A T$ activities

mRNA expression level of UCP1, Pparg,

Pgc1a, Prdm16, Cidea was significantly increased by PBA Treatment in primary cultured BAT cells

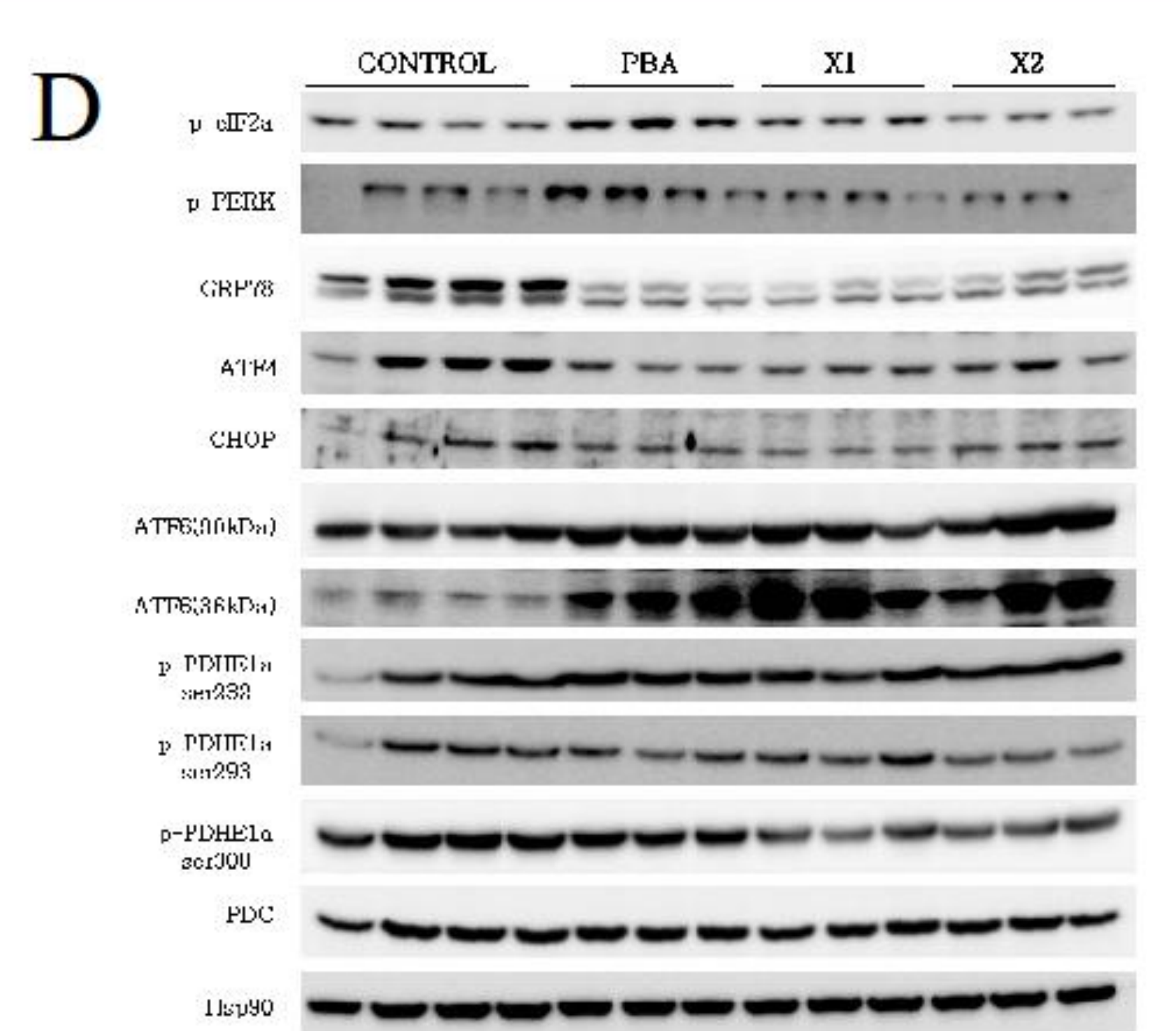

\section{CONCLUSION and REFERENCE}

1. $\mathrm{PBA}$ ameliorate obesity in $\mathrm{DIO}$ and $\mathrm{Ob} / \mathrm{Ob}$ mice model

2. PBA Increase BAT activities and Improved fatty liver and decrease macrophage infiltration in WAT

3. In liver tissue, PBA ameliorate ER stress, accompanied with decreased PDK activites

4. PDK may be therapeutic target to control ER stress induced in obese patients

\section{REFERENCE}

1. Science. 2006 Aug 25;313(5790):1137-40.

2. Sci Transl Med. 2013 Mar 6;5(175):175ra31.

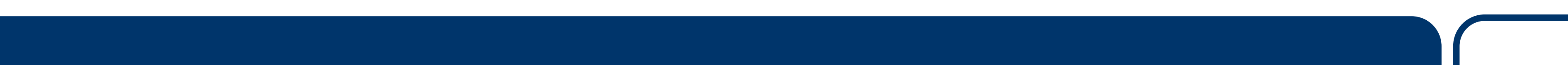

